\title{
Analysis of queries sent to PubMed at the point of care: Observation of search behaviour in a medical teaching hospital
}

\author{
Arjen Hoogendam*1,2, Anton FH Stalenhoef ${ }^{1}$, Pieter F de Vries Robbé ${ }^{2}$ and A \\ John PM Overbeke ${ }^{2}$
}

Address: ${ }^{1}$ Department of Medicine, Division of General Internal Medicine, Radboud University Nijmegen Medical Centre, Nijmegen, the Netherlands and 2Department of Medical Informatics, Radboud University Nijmegen Medical Centre, P.O. Box 9101, 6500 HB, Geert Grooteplein 21, Nijmegen, the Netherlands

Email: Arjen Hoogendam* - a.hoogendam@AIG.umcn.nl; Anton FH Stalenhoef - A.Stalenhoef@AIG.umcn.nl; Pieter F de Vries Robbé - P.deVriesRobbe@mi.umcn.nl; A John PM Overbeke - J.Overbeke@mi.umcn.nl

* Corresponding author

Published: 24 September 2008

BMC Medical Informatics and Decision Making 2008, 8:42 doi:10.1/86/1472-6947-8-42
Received: 13 May 2008

Accepted: 24 September 2008

This article is available from: http://www.biomedcentral.com/I472-6947/8/42

(C) 2008 Hoogendam et al; licensee BioMed Central Ltd.

This is an Open Access article distributed under the terms of the Creative Commons Attribution License (http://creativecommons.org/licenses/by/2.0), which permits unrestricted use, distribution, and reproduction in any medium, provided the original work is properly cited.

\begin{abstract}
Background: The use of PubMed to answer daily medical care questions is limited because it is challenging to retrieve a small set of relevant articles and time is restricted. Knowing what aspects of queries are likely to retrieve relevant articles can increase the effectiveness of PubMed searches. The objectives of our study were to identify queries that are likely to retrieve relevant articles by relating PubMed search techniques and tools to the number of articles retrieved and the selection of articles for further reading.
\end{abstract}

Methods: This was a prospective observational study of queries regarding patient-related problems sent to PubMed by residents and internists in internal medicine working in an Academic Medical Centre. We analyzed queries, search results, query tools (Mesh, Limits, wildcards, operators), selection of abstract and full-text for further reading, using a portal that mimics PubMed.

Results: PubMed was used to solve II2I patient-related problems, resulting in 3205 distinct queries. Abstracts were viewed in 999 (31\%) of these queries, and in 126 (39\%) of 32 I queries using query tools. The average term count per query was 2.5 . Abstracts were selected in more than $40 \%$ of queries using four or five terms, increasing to $63 \%$ if the use of four or five terms yielded 2-16I articles.

Conclusion: Queries sent to PubMed by physicians at our hospital during daily medical care contain fewer than three terms. Queries using four to five terms, retrieving less than $|6|$ article titles, are most likely to result in abstract viewing. PubMed search tools are used infrequently by our population and are less effective than the use of four or five terms. Methods to facilitate the formulation of precise queries, using more relevant terms, should be the focus of education and research. 


\section{Background}

Searching medical information on the internet has rapidly gained a place in daily medical care. Many sources are available for answering patient-centred questions. One of the main sources for medical information is Medline with PubMed as search engine. A major limitation of PubMed is that it takes 30 minutes on average to find information and appraise the literature critically [1]. When searching for patient-related problems at the point of care the physician wants to find information quickly $[2,3]$. Critical appraisal is the time-consuming step in the process. It is difficult to reduce the time needed to appraise the literature, which depends on the experience of the reader. However, reducing the number of articles that have to be appraised can reduce the search time significantly. It is difficult to retrieve only relevant articles from the large PubMed database as PubMed searches are characterised by retrieval of a vast number of article titles in very broad searches and a limited number of article titles in narrow searches [4]. The simplest method for reducing the numbers to read is to increase the number of terms in a query. Other PubMed tools available to the searching physician that can limit the number of retrieved articles are Boolean operators, Mesh and limits. A special set of tools advocated by evidence-based medicine handbooks [5,6], Clinical Queries, were designed to help in finding answers to clinical questions [7-12]. Many combinations of tools and term counts are possible and the results are often difficult to predict. As PubMed does not sort articles by relevance, the number of articles retrieved by a query plays a crucial role. Evaluation of hundreds of articles is useless when time is critical, but there is no information about the number of articles that can be scanned at the point of care. It is possible to issue several queries, increasing the accuracy of the query step by step, but this process is too timeconsuming for use during daily medical care. The physician should be able to find a potentially useful article within one or two queries, leaving enough time for critical appraisal. Observation of the search process during daily medical care is crucial for identifying the tools that actually work in this setting. We therefore created an online information portal that could monitor the complete search process without interfering with the search. Physicians working at our teaching hospital are accustomed to using online information sources and they have all received some education in evidence-based medicine. They are therefore likely to use a wide array of queries and search tools. We performed an observational study of queries sent to PubMed during daily medical care to answer the following questions. To what extent are search tools used, and does the use of these tools improve article selection for further reading? How many articles should be retrieved by a query to enhance the chance that one will be selected for further reading? What is the relationship between the number of terms, the articles retrieved by a query and abstract selection? We use abstract and full-text selection as parameters for success of a query.

\section{Methods}

\section{Population and measuring tool}

As part of an ongoing study of sources used for retrieving medical information we developed a web portal. This portal gives access to PubMed, two online medical textbooks (UpToDate, Harrison's Online) and a Dutch pharmacotherapy database. All residents and specialists in internal medicine selecting PubMed or UpToDate from our hospital information system were automatically linked to our portal.

\section{PubMed interface}

To enable all aspects of the use of PubMed to be registered we built our own interface that accesses PubMed through e-utils [13]. E-utils gives access to full PubMed functionality - queries are handled exactly as they are in the original PubMed website - but it delivers the data in XML, which allows them to be recorded in a database. The XML data need to be translated into web pages to be readable by users. To mimic the functionality of PubMed, most of the special search options relevant to patient-related searches $[5,6]$ were copied in our interface (Mesh database, details, a selection of limits (Publication date, publication types, human or animal and ages) and spelling (Figure 1). All queries were recorded along with the use of the different search options, the articles that were selected for abstract reading and the articles that were selected for full-text reading.

\section{Search process}

Every search was started by entering a query and selecting an information source. The sending of the first query about a problem was marked as the start of the search. During the search, all queries were recorded, as well as the database that was consulted. After the search was completed, the users were asked to select the situation that had led to the search (direct patient contact, patient rounds, scientific research, review/study, preparing talks, not specified).

\section{Query characteristics and evaluation of search result}

All queries sent to PubMed regarding patient-related problems (direct patient contact, patient rounds) were selected for analysis.

\section{Full-text and abstract selection as endpoints}

Queries resulting in the selection of abstracts and/or fulltext articles containing information that can be used to answer a question are considered as adequate queries that contribute to the search process. Ideally, the answer can be found in a single source by a single query, but in practice an answer to a question may be composed of multiple bits 

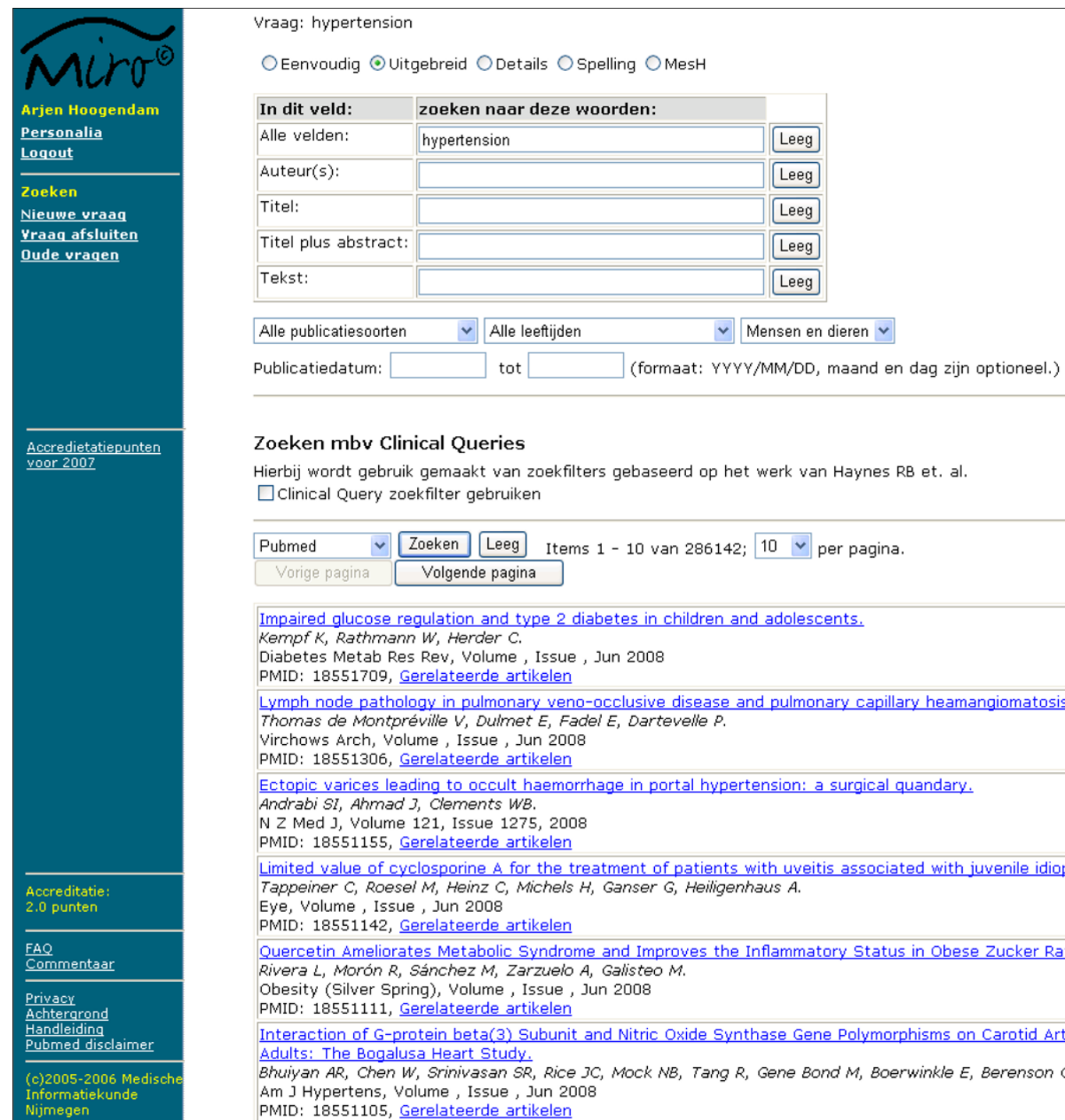

Zoeken mbv clinical Queries

Hierbij wordt gebruik gemaakt van zoekfilters gebaseerd op het werk van Haynes RB et. al.

$\square$ Clinical Query zoekfilter gebruiken

\begin{tabular}{|c|c|c|c|c|c|c|}
\hline Pubmed & $\checkmark$ & Zoeken & Leeg & Items 1 - 10 van 286142 & $10 \vee$ & per pagina. \\
\hline
\end{tabular}

Impaired glucose regulation and type 2 diabetes in children and adolescents.

Kempf $K$, Rathmann W, Herder $C$.

Diabetes Metab Res Rev, Volume, Issue, Jun 2008

PMID: 18551709 , Gerelateerde artikelen

Lymph node pathology in pulmonary veno-occlusive disease and pulmonary capillary heamangiomatosis.

Thomas de Montpréville V, Dulmet $E$, Fadel $E$, Dartevelle $P$.

Virchows Arch, Volume, Issue, Jun 2008

PMID: 18551306, Gerelateerde artikelen

Ectopic varices leading to occult haemorrhage in portal hypertension: a surgical quandary.

Andrabi SI, Ahmad' 3, Clements WB.

N Z Med J, volume 121, Issue 1275, 2008

PMID: 18551155, Gerelateerde artikelen

Limited value of cyclosporine $\mathrm{A}$ for the treatment of patients with uveitis associated with juvenile idiopathic arthritis.

Tappeiner C, Roesel M, Heinz C, Michels H, Ganser G, Heiligenhaus A.

Eye, Volume, Issue, Jun 2008

PMID: 18551142 , Gerelateerde artikelen

Quercetin Ameliorates Metabolic Syndrome and Improves the Inflammatory Status in Obese Zucker Rats.

Rivera L, Morón R, Sánchez M, Zarzuelo A, Galisteo M.

Obesity (Silver Spring), Volume, Issue, Jun 2008

PMID: 18551111, Gerelateerde artikelen

Interaction of G-protein beta(3) Subunit and Nitric Oxide Synthase Gene Polymorphisms on Carotid Artery Intima-media Thickness in Young Adults: The Bogalusa Heart Study.

Bhuiyan AR, Chen W, Srinivasan SR, Rice JC, Mock NB, Tang R, Gene Bond M, Boerwinkie E, Berenson GS.

A.m J Hypertens, Volume, Issue, Jun 2008

PMID: 18551105, Gerelateerde artikelen

\section{Figure I}

PubMed search interface. The advanced search options are available in the upper section. Besides search field descriptions (title, abstract and text word), several filters are available: publication types, age criteria, humans/animal and Clinical Queries filters. The PubMed search result for hypertension is shown in the lower section.

of information from several sources. Queries retrieving multiple articles that contain parts of the answer are therefore just as useful as queries that result in a single article containing the answer. The selection of an abstract containing information that contributes to the question is therefore a marker for the quality of the query. As the selection of abstracts is based on the title of articles some selected abstracts may not have attributed to the answer. This is a potential source of bias. Asking participants to rate the value of each selected abstract would result in interference with the search process. Participants would also refuse to use such an information source for an extended period. Interference with the search process is likely to result in bias, so the parameters of success of a query have to be extracted from search-related data. As it is unlikely that abstracts containing no information related to the question will be selected for full-text reading, selection of a full-text article is a marker for relevance of the abstract. However, not all abstracts contain links to full-text articles. Full text availability is therefore a possible confounder. Selection of irrelevant abstracts and online unavailability of full-text articles as sources of bias are unlikely to be related, as full-text availability does not depend on the relevance of the abstract to the question. If the results for full-text selection are comparable to those for abstract selection, both sources of bias are excluded. 
We therefore present data for both abstract and full-text selection.

\section{Relationship between number of terms and abstract selection}

PubMed combines all terms with the Boolean operator "AND". The use of terms without operators is therefore equivalent to combining all terms with the "AND" operator. Using more terms will therefore lead to fewer articles in the article result list. Most searches on the internet use only the "AND" operator, if any Boolean operator is used at all[14]. The number of articles retrieved by such a natural language query is directly related to the number and relevance of the terms used. To determine the relationship between the number of terms used in a query, the number of articles retrieved by a query and abstract selection we selected all queries containing natural language with or without the use of the "AND" operator. Queries containing the "OR" or "NOT" operator or Mesh terms were excluded. Terms were identified as words separated by a space. The "AND" operator was not counted as a term. The use of more than six terms in a query was too infrequent to merit detailed analysis. Evaluation of the relationship between term count and query result was therefore limited to queries containing fewer than seven terms. Terms that reflect the clinical question and are likely to retrieve relevant information are regarded as relevant in our study. Abstracts and full-text articles that contain information contributing to the question are considered relevant to the question.

\section{Relationship between terms, articles retrieved and abstract selection}

Only queries containing natural language that retrieved one or more articles were selected to demonstrate the relationship between term count and number of articles retrieved. Many terms will yield a small set of articles and a few terms will yield a large set. The number of articles retrieved by random terms therefore follows a logarithmic distribution. Combining several terms will not alter this distribution. As logarithmic numbers are difficult to interpret we divided the number of articles retrieved by a query into 14 equal intervals (average of 180 queries per category).

\section{Statistics}

Frequencies were used to summarize data. Significance was determined by the Chi-Square statistic using SPSS, release 14.0.2.

\section{Ethical approval}

No ethical approval was needed for this study, which involved no patients. All participants in our study consented to the use of search-related data for scientific research. Data were only collected if participants logged in at our internet portal.

\section{Results Query characteristics}

The use of PubMed was monitored from October 2005 until January 2007. During this period 3205 distinct queries were sent to PubMed. These queries were related to a total of 1121 patient-centred questions posed by 94 specialists and residents in internal medicine. In 999 (31\%) of the 3205 queries an abstract was selected for further reading (Table 1). In 456 (14\%), full-text was selected for further reading. The "AND" operator was frequently used, but as PubMed links all words in the query with "AND", the use of this operator is not necessary. Other operators, wildcards, Mesh or limits where used in $321(10 \%)$ of the 3205 queries. When these search tools were used, 126 $(39 \%)$ of 321 queries resulted in the selection of abstracts for further reading.

\section{Evaluation of the search result}

The query result is displayed as ten titles per page by default. To display more results, participants had to select the next page of results or change the number of articles displayed on screen. In 2625 (81.9\%) of the 3205 queries only the first ten titles were viewed and no consecutive pages were selected (table 2). In 1959 (61.1\%) of the queries, more than 10 articles were retrieved. Among these 1959 queries, only $20 \%$ of the retrieved articles were actually evaluated.

\section{Relationship between number of terms and abstract selection}

After selecting queries containing no Mesh, limits, wildcards or special operators ("AND" operator allowed), 2884 natural language queries remained. On average, 2.5 terms excluding operators were used in these queries. In 1617 (56\%) of the 2884 queries only 1 or 2 terms were

Table I: Aspects of queries sent to PubMed.

\begin{tabular}{ll}
\hline Aspects & All queries $(\mathrm{N}=3205)$ \\
\hline & $\mathrm{n}(\%)$ \\
\hline AND used * & $1409(44)$ \\
OR used * & $22(0.7)$ \\
NOT used * & $6(0.2)$ \\
wildcard used $\dagger$ & $65(2)$ \\
Mesh or Limits used $\ddagger$ & $252(8)$ \\
Query result positive $\S$ & $2521(79)$ \\
Abstract selected $\|$ & $999(31)$ \\
Full text selected $\|$ & $456(14)$ \\
\hline
\end{tabular}

\footnotetext{
* Boolean operators. † Asterisk functions as wildcard. $\ddagger$ All limits or Mesh terms were identified by the use of square brackets in a query. $\S$ One or more article titles retrieved by query. \| Queries that resulted in the selection of abstract or full-text articles for further reading.
} 
Table 2: Total number of titles that were displayed on screen by PubMed as a result of a query.

\begin{tabular}{ll}
\hline Titles* & Queries $(\mathrm{N}=3205)$ \\
\hline & $\mathrm{n}(\%) \dagger$ \\
\hline 10 & $2625(81.9)$ \\
20 & $284(8.9)$ \\
30 & $111(3.5)$ \\
40 & $62(1.9)$ \\
50 & $31(1.0)$ \\
$>50$ & $92(2.9)$ \\
\hline
\end{tabular}

*Total number of titles of articles in the query result list that were displayed on screen. If multiple pages of article titles were viewed for a single query the total number of pages presented on screen was calculated by adding the results displayed per screen. $\nmid$ Percentages do not add up to $100 \%$ because of rounding.

used, and $2828(98 \%)$ consisted of fewer than 6 terms (Figure 2). The relationship between the number of terms used and the proportion of queries leading to the selection of abstracts is shown in Table 3. Using more terms increases the risk of finding no articles at all. The percentage of queries yielding no articles slowly rises to $33 \%$ as the number of terms in a query rises to 6 . Increasing the number of terms in a query increases the proportion of queries leading to the selection of abstracts from 13\% (one term) to $43 \%$ (five terms). The proportion of queries leading to the selection of articles for full-text reading reaches a plateau of $23 \%$ when more than four terms are used.

\section{Relationship between terms, articles retrieved and abstract selection}

The percentage of queries resulting in abstract or full-text viewing as a function of the number of articles retrieved by a query is shown for 2521 queries that yielded one or more articles. The percentage of queries that led to abstract selection remains above $49 \%$ when $2-161$ articles are retrieved (Figure 3 ) and rapidly declines thereafter. The relationship between term count and abstract selection could be entirely attributable to the number of articles retrieved by a query. To determine the magnitude and dependence of each of these two parameters we looked at abstract selection in optimal queries for term count and/ or number of retrieved articles (table 4). These results show that retrieving 2-161 articles is a better predictor of abstract-viewing than using four to five terms in a query, but the two factors have independent effects as most queries lead to abstract selection if both conditions are met.

\section{Discussion}

Physicians at our university hospital, searching for patient-centred problems in PubMed, do not differ much from the general public using search engines such as Google[14]. They make very simple queries, containing two to three terms on average. In consequence, many queries yield a list of more than 161 articles, which are not further evaluated for relevance. The use of PubMed search tools was very limited and the performance of these tools was comparable to the use of more than three terms in a query.

\section{Query characteristics}

Our participants used two to three terms on average. Previous research found three terms [15]; this difference may be because we did not count Boolean operators as terms, unlike the authors of [15]. As all searches are connected to patient-related problems we expected the queries to contain more terms to describe the question more adequately. Another reason for expecting more terms in a query is that general questions are relatively easy to find in information sources containing aggregated data, such as evidencebased textbooks. Physicians are therefore advised to use reviews and studies as consecutive last steps in the search process when other sources cannot provide an answer [16]. This makes it unlikely that the questions that were looked up in PubMed were general in nature. The more likely reason for lack of detail is that despite all recommendations for constructing proper queries in evidencebased medicine $[5,6]$, physicians do not take the time to construct such queries. A study by Ely et al showed that physicians could not answer $41 \%$ of pursued questions. Analysis of unanswered questions showed that it was possible to answer a proportion of unanswered questions if queries were reformulated, better describing the question $[17,18]$. It has been shown that training courses in evidence-based practice improve search skills considerably $[19,20]$. Our results show that term count and number of retrieved articles in the query result have independent effects. If using more terms only reduced the number of irrelevant articles, then term count should not have an independent effect. Using more terms related to a question must therefore also increase the number of relevant articles. This is most likely to be related to a more precise description of the question. Although the percentage of queries yielding no articles rises slowly with the use of more terms, it does not have a negative effect on abstract selection up to at least 6 terms. Physicians should therefore be urged to use enough terms, describing the question accurately, and should not fear that this will yield too few articles. As our population is familiar with evidencebased searching, the question is why they do not use advanced search methods. One possible reason is that search tools are not on the main page of our portal and PubMed but require navigation to special search sections. As truly effective tools are likely to be used even when they are difficult to locate, this may not be a valid argument. Another reason might be that participants do not use the PubMed search tools effectively. Our participants selected fewer abstracts with search tools than with the use of four 


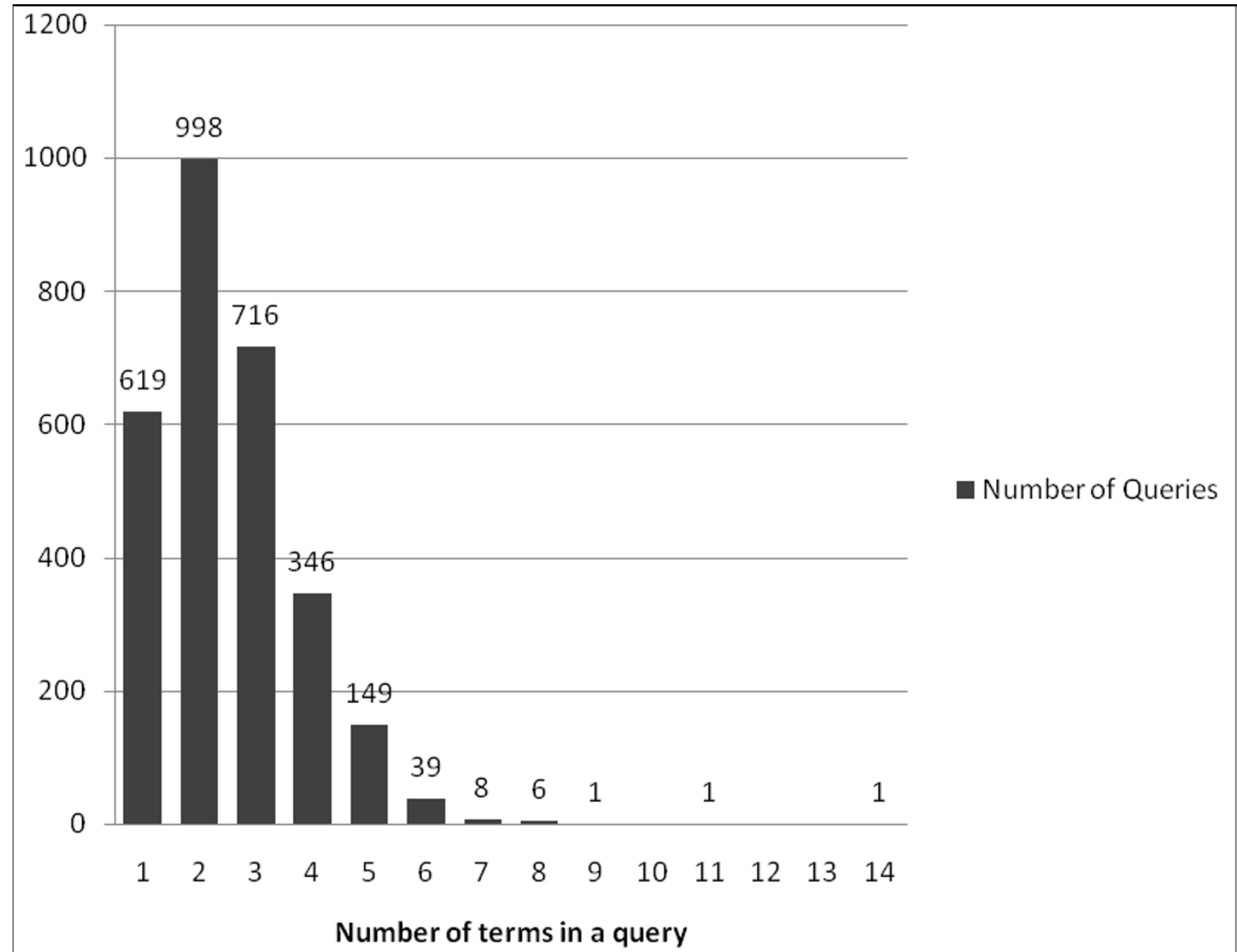

Figure 2

Distribution of term count in PubMed queries. Selection of 2884 queries containing no Mesh headings, limits, wildcards or special operators, "AND" operator allowed.

or five terms, and this might be related to improper use of the search tools. Tools that are effective in laboratory situations but are difficult to use properly during daily medical practice are inefficient for this type of search and should not be advocated for use initially. A final reason might be that other search engines do not require the use of advanced search methods and physicians try to search in the way most familiar to them. Examples of such search engines, delivering ranked results, are Google, Google Scholar and Relemed [21]. Because these search engines perform relevance ranking they can be used effectively with natural language queries. The relative ease of Google searching has led to a publication advocating the use of Google to help solve patient-related diagnostic problems [22]. The question is whether physicians should be taught to use these search engines or to use better search tech- niques in PubMed. One argument against Google is that there are several fundamental issues regarding the reliability of the information retrieved and the validity of the ranking method [23]. More importantly, formulating accurate clinical questions and translating them into well formed queries, with or without the use of additional search tools, is likely to increase the accuracy of the search result regardless of the search engine used.

\section{PICO as a method to improve a query}

One method for translating clinical questions into accurate queries is the PICO method. This method can help to build adequate queries regarding patient-related problems $[5,6,24,25]$. In the PICO method the physician is instructed to describe the patient-related problem in three to four concepts (Patient characteristics, Intervention, 
Table 3: Queries that yielded no articles in the PubMed result list, queries that resulted in abstract selection and queries that resulted in full-text selection in relation to the number of terms used.

\begin{tabular}{llll}
\hline \multirow{2}{*}{ Terms } & Query result & & \\
\cline { 2 - 4 } & No articles retrieved by query & Abstract selected & Full-text selected \\
\hline & $\mathrm{n} / \mathrm{N}(\%)$ & $\mathrm{n} / \mathrm{N}(\%)$ & $\mathrm{n} / \mathrm{N}(\%)$ \\
\hline 1 & $101 / 619(16)$ & $79 / 619(13)$ & $28 / 619(5)$ \\
2 & $197 / 998(20)$ & $291 / 998(29)$ & $108 / 998(11)$ \\
3 & $174 / 716(24)$ & $277 / 716(39)$ & $80 / 346(23)$ \\
4 & $86 / 346(25)$ & $145 / 346(42)$ & $32 / 149(21)$ \\
5 & $42 / 149(28)$ & $64 / 149(43)$ & $9 / 39(23)$ \\
\hline
\end{tabular}

Selection of 2867 queries containing no Mesh headings, limits, wildcards or special operators, "AND" operator allowed. Queries containing more than 6 terms excluded.

Comparison and Outcome). This technique was designed for questions regarding therapy but can be adapted to questions about diagnosis. Using the PICO formulation is likely to result in better queries, limiting the number of results. Although the majority of questions posed by clinicians are related to treatment and diagnosis that can be translated into PICO, many clinical questions cannot be translated into PICO. For example, questions regarding prognosis, the etiology of a disease, economic consequences, biochemical compounds, physiological principles, pathology, genetics and complications are difficult to translate. This is one of the limitations of PICO. Hersk-

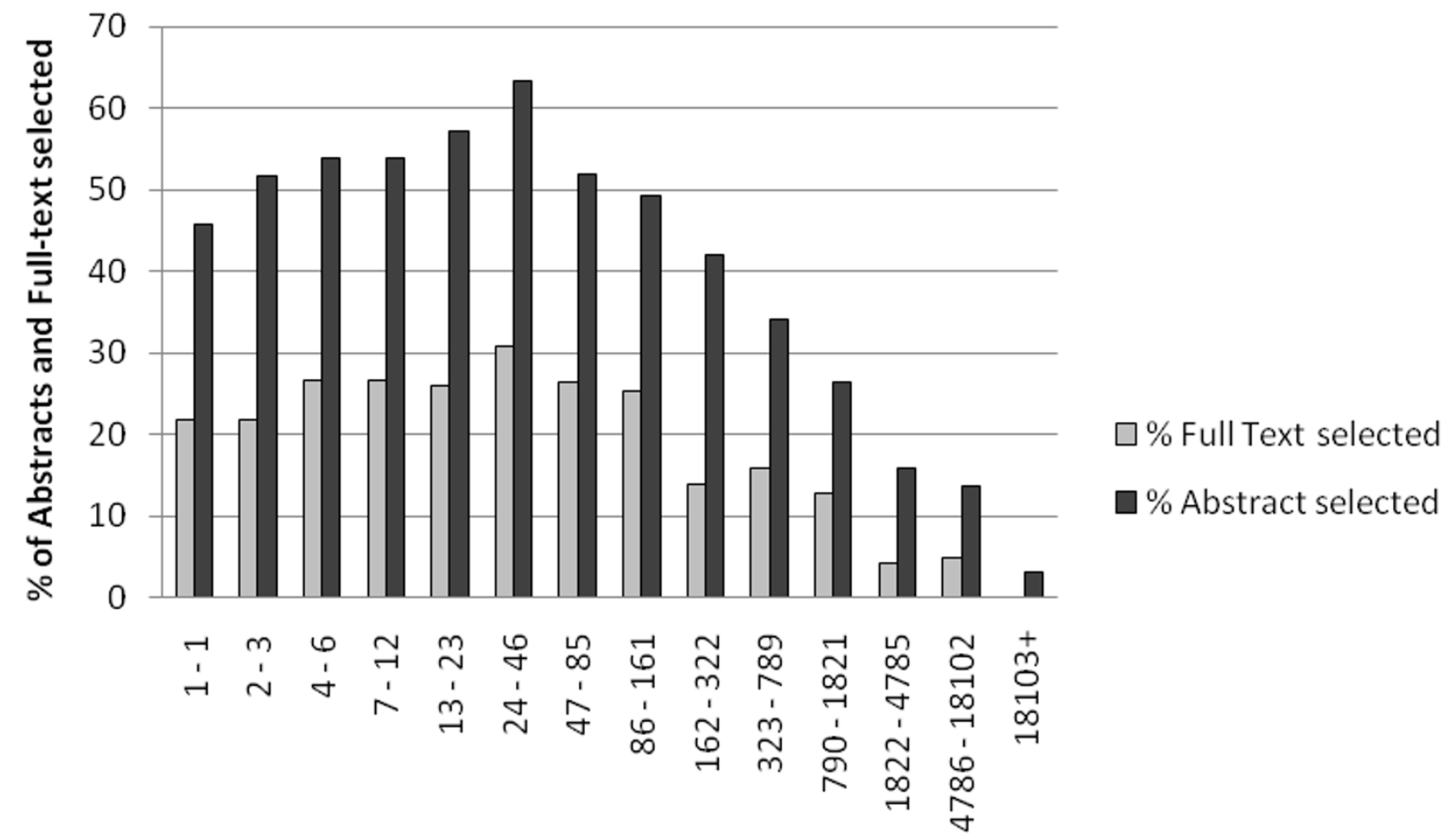

\section{Number of articles retrieved by the query}

Figure 3

Percentage of queries leading to abstract or full-text reading in relation the number of articles retrieved by a query. Selection of 2521 queries that yielded one or more articles. 
Table 4: Relationship between optimal term count and optimal number of articles retrieved by a query, cross-tabulated by abstract selection.

\begin{tabular}{|c|c|c|c|}
\hline & 4 or 5 Terms & Fewer or more than 4 or 5 Terms & \\
\hline & $\mathrm{AS} / \mathrm{NQ}(\%) \dagger$ & AS/NQ(\%)† & NQt \\
\hline $2-161$ articles retrieved by query & $161 / 254(63)$ & $4 I I / 807(5 I)$ & 1061 \\
\hline I or more than 161 articles retrieved & $48 / 113(42)$ & $248 / 1080(23)$ & 1193 \\
\hline Total NQ & 367 & 1887 & 2254 \\
\hline
\end{tabular}

Selection of 2254 queries resulting in one or more retrieved articles and containing no Mesh headings, limits, wildcards or special operators, "AND" operator allowed. $†$ AS $=$ Queries leading to Abstract Selection. NQ $=$ Total Number of Queries in category.

ovic et al. stated that educators and PubMed user interface researchers should not focus on specific topics, but on overall efficient use of the system [15]. It is not feasible or practical to create versions of PICO adapted for all possible medical questions. As PICO is a method to break down a question into several concepts it might be useful to break down the question into several concepts regardless of the topic. We show that creating a PubMed query using four or five relevant terms is a good option to start with, regardless of the search topic. Using search tools may increase the search results further but we could not prove this because of the limited use of advanced search tools.

\section{Abstract selection in relation to query evaluation, retrieved articles and terms}

The number of articles retrieved by a query showed a nearly logarithmic distribution, comparable to previous results[15]. The fact that rarely more than the first ten results were evaluated is an important finding. Previous research has shown that searchers seldom view more than 20 results when using search engines with relevance ranking[14]. Because such engines are likely to display the most relevant results on the first page, this can be a reasonable strategy. PubMed, however, does not perform relevance ranking, but by default displays the articles roughly by publication date in PubMed, beginning with the most recent. It is also possible to sort articles by author, actual publication date, journal and title but not according to relevance to the query. The chance of finding a relevant abstract within a list of several hundreds of article titles sorted by publication date, when only a fraction of the result is reviewed, is very low. Given the number of articles viewed on average by our population, the percentage of queries resulting in abstract selection started to decline rapidly with queries yielding more than 161 articles. The number of articles retrieved by a query is influenced by the number of terms used. Although using more relevant terms will usually result in a more accurate search result, using more terms increases the risk that the query will yield no results or no relevant results. The decline in the number of abstracts viewed when more than 5 terms are used can be explained by this phenomenon. The ques- tion is whether the fact that 4 or 5 terms in a query are optimal can be wholly attributed to the number of articles retrieved by a query. As both term count and number of articles retrieved affect the viewing of abstracts, one factor cannot be attributed entirely to the other.

\section{The query in relation to the search process}

We investigated single queries, but the entire search process usually consists of sequential steps that should lead to an answer. After a PubMed query retrieves a set of articles the searcher may choose to evaluate a certain percentage of the abstracts and full-texts, but may also decide to refine the query. If the result is too large the query may be refined using hedges or more terms. If the result is too small the searcher may choose to remove terms that are too specific or expand terms with the "or" operator. The effects of these different measures are difficult to predict, especially if several options are combined. It is not surprising that using more relevant terms in a query will lead to fewer articles in the result, increasing the chance of article evaluation. The fact that four or five terms were optimal and fewer than 161 articles were optimal was an important finding. A previous study, describing the implementation of a Medline search tool for handhelds in a clinical setting, reported optimal values for term count and retrieved articles comparable to our results [26]. Knowing the optimal values can help in the design of search interfaces that promote the use of multiple terms in a query and the use of search tools, but can also aim for an optimal number of retrieved articles. Presenting the first ten unsorted results of several thousand articles is not useful for searching physicians. Analysis of queries that did not retrieve a sensible number of articles can help to guide the physician to increase the accuracy of the query, thus increasing the chance of retrieving a reasonable number of articles.

\section{Limitations}

We observed Dutch physicians. As English is not their native language they may have used erroneous terms, which is likely to result in more queries with no articles in the result. 
A possible source of error is that PubMed is our default database for searching. If a physician entered a query for UpToDate but forgot to select UpToDate as the search database, the query was sent to PubMed. Sending a query containing one term to other databases is usually sufficient, so the number of single term queries sent to PubMed might have been overestimated.

Our observation that the effect of using Mesh and limits is comparable to that of using adequate terms in a query is consistent with previous research $[27,28]$.

We treated all queries as single entities and did not focus on the process of refining them. There is no way that a previous query can influence the articles retrieved by the next, so it cannot influence the next query result. Article selection might depend on experience from previous queries. Articles that were scanned in the first query will not be scanned in the second regardless of relevance to the question, so selection of articles in previous queries is not likely to result in bias.

Because we have observed natural behaviour by physicians in a very specific setting, our results are likely to be influenced by many factors and different ones may be obtained in different settings, limiting their generalizability.

\section{Conclusion}

Our study is new in performing a detailed observation of the PubMed search process during busy medical practice in a hospital setting. Physicians at our hospital make very simple queries, containing fewer than three terms, and $31 \%$ result in viewing of abstracts. Search tools increased the selection of abstracts moderately to $39 \%$. Both term use and number of retrieved articles influence abstract selection. Queries containing four or five terms yielding 2-161 articles were most effective in our population, with $63 \%$ abstract-viewing. PICO and other methods for improving query formulation should be the focus of more research and teaching, as this is likely to help considerably in improving search results during daily medical practice. Search engines aimed at on-the spot searching should analyze queries and give advice how to improve queries that retrieve too few or too many results instead of displaying the titles of the articles retrieved.

\section{Competing interests}

The authors declare that they have no competing interests.

\section{Authors' contributions}

$\mathrm{AH}, \mathrm{PV}, \mathrm{AS}$ and $\mathrm{AO}$ conceived and designed the study. The design of the internet portal was coordinated by $\mathrm{AH}$ and supervised by PV and AO. AH was responsible for data acquisition, interpretation and analysis. AH drafted the manuscript. All authors were involved in its critical revision and final approval for publication.

\section{Acknowledgements}

The authors thank the residents and specialists in internal medicine who participated in the study. We also thank Egbert van der Haring and Pieter Zanstra for the design of the portal and advice on analysis.

\section{Role of the funding source}

This study was funded by the Vereniging Nederlands Tijdschrift voor Geneeskunde (Association Dutch Journal of Medicine). This Association was not involved in the design and conduct of the study; collection, management, analysis, or interpretation of the data; or in the preparation, review or approval of the manuscript.

\section{References}

I. Hersh WR, Hickam DH: How well do physicians use electronic information retrieval systems? A framework for investigation and systematic review. JAMA 1998, 280:| 347-I 352.

2. Coumou HC, Meijman FJ: How do primary care physicians seek answers to clinical questions? A literature review. J Med Libr Assoc 2006, 94:55-60.

3. Schwartz K, Northrup J, Israel N, Crowell K, Lauder N, Neale AV: Use of on-line evidence-based resources at the point of care. Fam Med 2003, 35:25I-256.

4. Davidoff $F$, Florance $V$ : The informationist: a new health profession? Ann Intern Med 2000, 132:996-998.

5. Straus SE, Richardson WS, Glasziou P, Haynes RB: Evidence-based Medicine: How to Practice and Teach EBM Edinburgh: Churchill Livingstone; 2005

6. Guyatt GH, Rennie D: Users' Guides to the Medical Literature: A Manual for Evidence-Based Clinical Practice (Users' Guides to the Medical Literature: a Manual for Evidence-Based Clinical Practice) American Medical Association Press; 2002.

7. Haynes RB, Wilczynski NL: Optimal search strategies for retrieving scientifically strong studies of diagnosis from Medline: analytical survey. BMJ 2004, 328: 1040.

8. Haynes RB, McKibbon KA, Wilczynski NL, Walter SD, Werre SR: Optimal search strategies for retrieving scientifically strong studies of treatment from Medline: analytical survey. BMJ 2005, 330: II 79.

9. Montori VM, Wilczynski NL, Morgan D, Haynes RB: Optimal search strategies for retrieving systematic reviews from Medline: analytical survey. BMJ 2005, 330:68.

10. Wilczynski NL, Haynes RB, Lavis JN, Ramkissoonsingh R, Arnold-Oatley $A E$ : Optimal search strategies for detecting health services research studies in MEDLINE. CMAJ 2004, I 7 I: I I79- I I 85.

1I. Wilczynski NL, Haynes RB: Developing optimal search strategies for detecting clinically sound prognostic studies in MEDLINE: an analytic survey. BMC Med 2004, 2:23.

12. Wong SS, Wilczynski NL, Haynes RB: Developing optimal search strategies for detecting clinically relevant qualitative studies in MEDLINE. Stud Health Technol Inform 2004, I07(Pt I):3 I I-3 I6.

13. Entrez utilities [http://eutils.ncbi.nlm.nih.gov/entrez/query/static/ eutils help.html]

14. Jansen BJ: The effect of query complexity on Web searching results. Information Research 2000, 6:.

15. Herskovic JR, Tanaka LY, Hersh W, Bernstam EV: A day in the life of PubMed: analysis of a typical day's query log. J Am Med Inform Assoc 2007, 14:212-220.

16. Haynes RB: Of studies, syntheses, synopses, summaries, and systems: the " $5 \mathrm{~S}$ " evolution of information services for evidence-based healthcare decisions. Evid Based Med 2006, II:162-164.

17. Ely JW, Osheroff JA, Maviglia SM, Rosenbaum ME: Patient-care questions that physicians are unable to answer. J $\mathrm{Am}$ Med Inform Assoc 2007, I 4:407-4I 4.

18. Ely JW, Osheroff JA, Chambliss ML, Ebell MH, Rosenbaum ME Answering physicians' clinical questions: obstacles and potential solutions. J Am Med Inform Assoc 2005, 12:217-224. 
19. Smith CA, Ganschow PS, Reilly BM, Evans AT, McNutt RA, Osei A, Saquib M, Surabhi S, Yadav S: Teaching residents evidence-based medicine skills: a controlled trial of effectiveness and assessment of durability. J Gen Intern Med 2000, I 5:7 I 0-7I 5.

20. Cabell CH, Schardt C, Sanders L, Corey GR, Keitz SA: Resident utilization of information technology. J Gen Intern Med 200I, 16:838-844.

21. Siadaty MS, Shu J, Knaus WA: Relemed: sentence-level search engine with relevance score for the MEDLINE database of biomedical articles. BMC Med Inform Decis Mak 2007, 7:I.

22. Tang $\mathrm{H}, \mathrm{Ng} \mathrm{JH}$ : Googling for a diagnosis - use of Google as a diagnostic aid: internet based study. BM/ 2006, 333: | | 43- | | 45.

23. Grivell L: Seek and you shall find? EMBO Rep 2006, 7:I0-I3.

24. Huang X, Lin J, Demner-Fushman D: Evaluation of PICO as a knowledge representation for clinical questions. AMIA Annu Symp Proc 2006:359-363.

25. Schardt C, Adams MB, Owens T, Keitz S, Fontelo P: Utilization of the PICO framework to improve searching PubMed for clinical questions. BMC Med Inform Decis Mak 2007, 7:16.

26. Hauser SE, Demner-Fushman D, Jacobs JL, Humphrey SM, Ford G, Thoma GR: Using wireless handheld computers to seek information at the point of care: an evaluation by clinicians. J Am Med Inform Assoc 2007, 14:807-8I5.

27. Jenuwine ES, Floyd JA: Comparison of Medical Subject Headings and text-word searches in MEDLINE to retrieve studies on sleep in healthy individuals. J Med Libr Assoc 2004, 92:349-353.

28. Hersh W, Hickam D: Use of a multi-application computer workstation in a clinical setting. Bull Med Libr Assoc 1994, 82:382-389.

\section{Pre-publication history}

The pre-publication history for this paper can be accessed here:

http://www.biomedcentral.com/1472-6947/8/42/prepub

Publish with Biomed Central and every scientist can read your work free of charge

"BioMed Central will be the most significant development for disseminating the results of biomedical research in our lifetime. "

Sir Paul Nurse, Cancer Research UK

Your research papers will be:

- available free of charge to the entire biomedical community

- peer reviewed and published immediately upon acceptance

- cited in PubMed and archived on PubMed Central

- yours - you keep the copyright

Submit your manuscript here:

http://www.biomedcentral.com/info/publishing_adv.asp
BioMedcentral 